\title{
Robotic Surgery in Otolaryngology: Endocrine
}

\author{
Salem I. Noureldine $\cdot$ Jeremy D. Richmon • \\ Ralph P. Tufano $\cdot$ Salah Mohamed • \\ Emad Kandil
}

Published online: 24 July 2013

(C) Springer Science+Business Media New York 2013

\begin{abstract}
Over the last decade, robotic surgery has evolved from a medical curiosity, with anticipated potential to overcome the limitations of surgical endoscopy, to the favored procedure in several surgical specialties such as urology and gynecology. The application of robotic technology in head and neck endocrine surgery has recently expanded. Different approaches have lately been described that are intended to preserve the safety of these operations, while avoiding visible neck scars. This article aims to provide the reader with an overview of the current robotic approaches and their capability to assist surgeons in accomplishing remote-access thyroid and parathyroid surgery.
\end{abstract}

Keywords Robotic thyroidectomy $\cdot$ Robotic parathyroidectomy · Targeted parathyroidectomy · Remote access $\cdot$ Transaxillary $\cdot$ Post-auricular

Electronic supplementary material The online version of this article (doi:10.1007/s40136-013-0024-7) contains supplementary material, which is available to authorized users.

S. I. Noureldine $\cdot$ J. D. Richmon · R. P. Tufano

Division of Head and Neck Endocrine Surgery, Department of Otolaryngology-Head and Neck Surgery, The Johns Hopkins

University School of Medicine, Baltimore, MD, USA

S. Mohamed · E. Kandil ( $\square)$

Endocrine Surgery Section, Edward G. Schlieder Chair in Surgical Oncology, Division of Endocrine and Oncological Surgery, Department of Surgery, Tulane University School of Medicine, 1430 Tulane Avenue, Room 8510 (Box SL-22), New Orleans, LA 70112, USA

e-mail: ekandil@tulane.edu

\section{Introduction}

Traditional thyroid and parathyroid surgery is usually performed through a skin crease incision in the anterior neck, requiring the creation of myocutaneous flaps to gain exposure to the thyroid compartment. This facilitates a safe dissection and is associated with low morbidity in experienced hands. However, some patients are still left with a conspicuous cervical scar. Most of this population comprises young women who are understandably concerned about preserving their neck appearance and avoiding visible neck scars. As a result, there has been a great desire among both surgeons and patients to minimize surgical incisions or relocate them outside the neck so that they are "invisible" incisions. Endoscopic techniques evolved first in the field of parathyroid surgery [1•]. Significant improvements in endoscopic instrumentation, preoperative localization studies, and the increased understanding of the endoscopic cervical anatomy have facilitated the further growth of head and neck endoscopic surgery for the management of thyroid and parathyroid disease [2-9].

Since its introduction in the 1990s, robotic surgery has evolved from a novelty, with anticipated potential to overcome the limitations of endoscopic surgery, to the favored approach in several surgical disciplines such as urology [10], gynecology [11], and cardiothoracic surgery [12-16•]. The evolution of robotic head and neck surgery has expanded on the earlier achievements of endoscopic surgery for thyroid and parathyroid disease. Different robotic approaches have lately been described that are intended to avoid cervical scarring, but also result in less pain with a faster return to functional activities as documented in the Korean experience [17, 18••, 19••, 20, 21]. Surgeons have found that the ability to control a magnifying three-dimensional high-definition camera system 
with a stable platform and multiarticulated tremor-free endoscopic arms through a single console restores some of the fundamentals that were lost in the transition to endoscopic surgery. This is particularly advantageous in the restricted workspace afforded in this region of the body $[8,20,22]$.

This article aims to provide the reader with an overview of the current robotic approaches used in robotic thyroid and parathyroid surgery. Today's patients seek out surgeons who can and will offer these novel options when appropriate. Surgeons should therefore be facile in each of these techniques so that each approach can be individualized on the basis of any given situation.

\section{Robotic Parathyroidectomy}

\section{Background}

Historically until the 1990s, bilateral cervical exploration of all four parathyroid glands and removal of any that are grossly enlarged was the standard surgical treatment for primary hyperparathyroidism. In the past two decades, significant improvements in the accuracy and reliability of preoperative localization studies have facilitated further evolution in surgical management, allowing a more targeted minimally invasive surgical approach [23]. Because 80-90\% of patients with primary hyperparathyroidism have a solitary parathyroid adenoma, resection of one gland leads to cure in most cases. The first unilateral approach for solitary parathyroid adenoma resection was reported by Tibblin et al. [24•]. Since then, several targeted techniques have been described, including radio-guided parathyroidectomy, endoscopic parathyroidectomy with gas insufflation, and video-assisted parathyroidectomy without gas insufflation $[1 \bullet, 25,26]$.

As technology and training have advanced, the da Vinci robotic surgical system (Intuitive Surgical, Sunnyvale, CA, USA) has recently evolved as an adjunct in parathyroid surgery. Robotic parathyroidectomy has been described lately by us and other groups in a few case reports and small series using an axillary incision [21, 27-31]. The robotic transaxillary approach permits a safe, precise, magnified dissection without the need for gas insufflation and has a better cosmetic result owing to the invisible scar in the neutral position. However, it should be emphasized that this remote access approach is not considered "minimally invasive," as it actually requires much more dissection than the traditional endoscopic parathyroidectomy. A robotic-assisted thoracoscopic approach has also been reported for parathyroid adenomas located within the mediastinum [32-34].
Indications and Patient Selection

Robotic parathyroidectomy takes advantage of the endoscopic magnification that allows the same intervention to be performed through a remote, so-called scarless access. Nonetheless, ideal patient selection criteria are not well established. The best candidates for this approach are small or average-sized (body mass index below $30 \mathrm{~kg} / \mathrm{m}^{2}$ ) young patients, with concerns of neck scarring, or who have a history of keloid or hypertrophic scar formation. This approach should only be offered to patients with a welllocalized parathyroid adenoma preoperatively on parathyroid scans and ultrasound neck examinations. Patients with greater possibility of multiglandular disease should not be offered this approach. Use of this approach is usually deferred in patients with a previous history of neck surgery or irradiation of the neck. Patients should also be screened for contraindications that affect patient positioning during this procedure, such as rotator cuff disease, shoulder/neck mobility problems, cervical spine disease, and previous neck, chest, or axillary surgery.

\section{Surgical Planning}

Imaging before surgery will help guide the surgical approach by localizing the adenoma in many patients. To further improve the surgical success of targeted parathyroidectomy and to minimize the possibility of persistent or recurrent hyperparathyroidism after surgery, some have advocated the use of surgical adjuncts such as intraoperative parathyroid hormone (PTH) monitoring. This is useful in assessing the adequacy of resection by functional means without the need for bilateral neck exploration. The ability to confirm complete removal of all hypersecreting glands and predict operative success minimized operative time, diminished the need for bilateral neck exploration, and improved cure rates [35].

\section{Surgical Technique}

We routinely perform intraoperative ultrasound examination after intubation and prior to surgical incision. This provides reinforcement of the parathyroid adenoma location in relation to the thyroid lobe and the relation to the nearby vital structures, including the carotid sheath and esophagus. The surgical procedure includes flap elevation under direct vision to make a working space, docking the robot system, and console work. We routinely perform continuous nerve monitoring of the ulnar, radial, and median nerves to avoid neuropraxia [36••]. We also use continuous intraoperative nerve stimulation to definitively identify nerve structures during the procedure. The patient is placed in a supine position with a gently extended neck, 
and the arm ipsilateral to the lesion is extended at the shoulder and flexed at the elbow. This optimizes exposure of the axilla and creates a short distance from the axillary skin to the thyroid gland, through which dissection will be performed. Blood is drawn to obtain a baseline rapid PTH serum level prior to preparing the neck or palpating the neck. A 2-in. incision is then made in the anterior axillary line. Attention to detail in incising and handling the skin reduces cicatrix hypertrophy. The subcutaneous flap is raised in the direction of the thyroid until the clavicle. The neck platysma is then incised and the plane between the sternal and clavicular heads of the sternocleidomastoid muscle is visualized and developed with cautery or the Harmonic scalpel. A lightened retractor is used to elevate the sternal head, exposing the strap muscles. The natural dehiscence between the sternal and clavicular heads is entered, allowing identification of the carotid sheath and ipsilateral omohyoid and sternohyoid muscles. The strap muscles are then elevated off the thyroid gland, providing exposure from the sternal notch to the superior pole and across the midline. A wound protector is placed to protect the axillary wound edges from any heat generated by the electocautery or the Harmonic scalpel. A specially designed retractor is placed under the sternal head of the sternocleidomastoid and strap muscles and is secured to the other side of the operating room bed to maintain an adequate working space without gas insufflation. The robot is then docked from the side of the bed contralateral to the operative field. The robotic instruments used are the ProGrasp forceps (Intuitive Surgical, Sunnyvale, CA, USA), the Maryland dissector (Intuitive Surgical, Sunnyvale, CA, USA) and the Harmonic scalpel (Ethicon, Somerville, NJ, USA). The $30^{\circ}$ endoscope is used in a downward facing orientation. The Maryland dissector and Harmonic scalpel should be as far apart as possible. This is important in minimizing the risk of collision of the arms during the procedure. The assistant uses a laparoscopic suction irrigator via the axillary incision. The thyroid gland is turned medially, and with cautious dissection the pathological parathyroid gland is identified. The middle thyroid vein is divided using the Harmonic scalpel. Identification of the inferior thyroid pedicle with dissection of the recurrent laryngeal nerve in the tracheoesophageal groove is then undertaken to minimize the risk of injury to either structure. A nerve stimulator is routinely used by the assistant surgeon to confirm identification of the recurrent laryngeal nerve. Once the pedicle has been delineated, the Harmonic scalpel is used to hemostatically seal and divide the small branches of the inferior thyroid artery close to the capsule of the adenoma. The parathyroid lesion is then dissected, excised, and delivered through the axillary incision (Fig. 1, Video S1). After gland removal, a serum sample is drawn for rapid PTH analysis. A $50 \%$ or greater drop in PTH
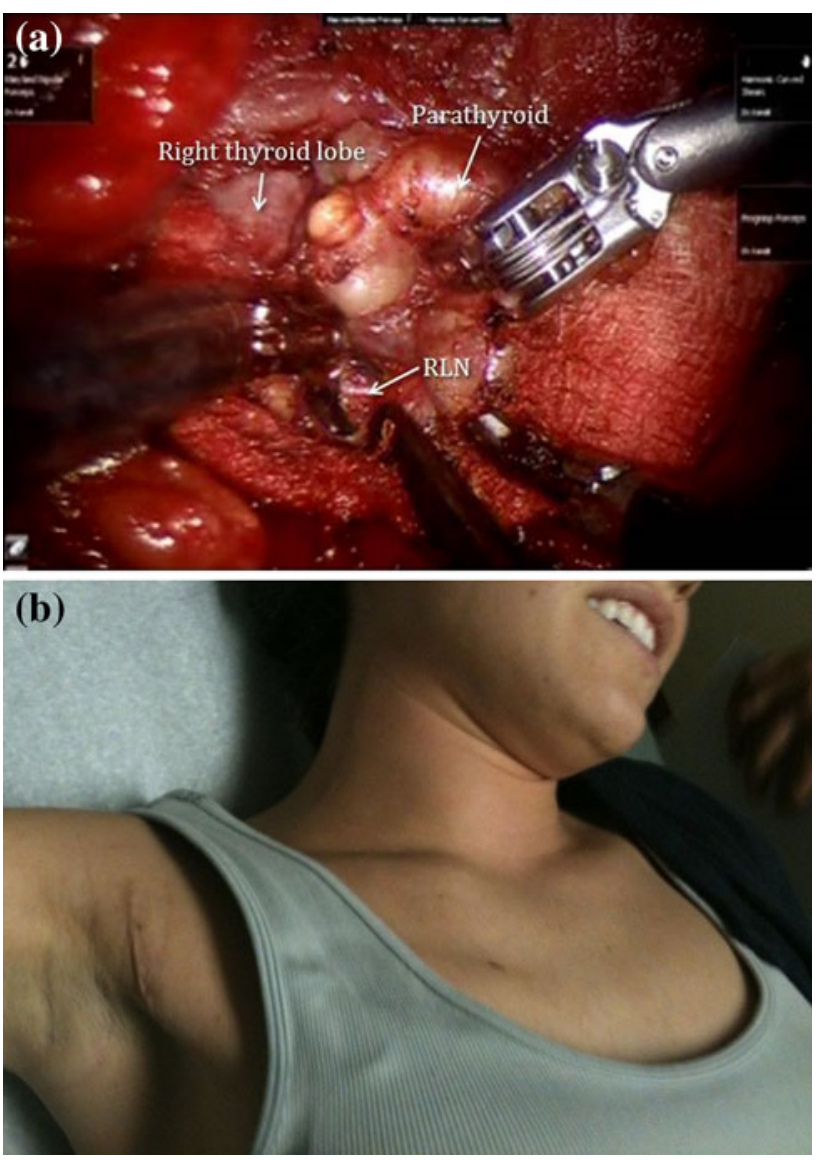

Fig. 1 The robotic technology affords a true three-dimensional view of the surgical field, facilitating the identification of the diseased parathyroid gland and the recurrent laryngeal nerve $(R L N)$ in the tracheoesophageal groove (a). The cosmetic results are superior (b) and valued by patients undergoing this approach

level and to within the reference range predicts a successful single-gland surgery. The incision is then closed; however, the patient is kept sedated and the surgical field is maintained until the laboratory results are received. Those patients with no change in PTH level or inadequate reduction of the PTH level likely have a secondary adenoma (or less likely an unappreciated multiple endocrine neoplasia syndrome).

Robotic transaxillary parathyroidectomy is performed as an outpatient procedure. The patients are discharged in receipt of anti-inflammatory pain medication, with narcotics only for breakthrough discomfort. Patients are supplemented with $0.25 \mathrm{mg}$ calcitriol twice daily and $1 \mathrm{~g}$ elemental calcium twice daily unless signs or symptoms of hypocalcemia are present. No laboratory studies are required following intraoperative verification of normalization serum PTH level. The patient's first outpatient follow-up is at 1 week for wound inspection and vocal fold examination. 


\section{Complications}

All the reports of robotic parathyroidectomy have shown it to be safe, feasible, and efficacious. Complications such as recurrent laryngeal nerve palsy and hypoparathyroidism are rare (less than $1 \%$ ), similarly to the conventional cervical approach. The preliminary functional outcomes of robotic parathyroidectomy are encouraging. Long-term prospective outcome data are imminent and randomized clinical studies are warranted to evaluate potential advantages. There is little published in the literature which evaluates the conversion rates associated with robotic parathyroid surgery [37]. Nonetheless, conversion to a wider access or conventional procedure for bilateral neck exploration should not be considered a complication; it is a limitation of the preoperative localization studies and focused surgical approach rather than a reflection of the robotic technique per se. Nevertheless, a high conversion rate may reflect poor patient selection. Additionally, prolonged paresthesia under the flap and muscle stiffness have been described by some patients. All patients should be counseled preoperatively regarding this matter. The arm positioning can cause overtraction and brachial plexus neuropraxia. Use of somatosensory evoked potential response nerve monitoring should help avoid these complications.

\section{Robotic Thyroidectomy}

\section{Background}

Thyroid surgery has an ancient history, dating back to the early sixth century A.D., of being predominantly used for the treatment of thyroid goiters causing obstructive symptoms. The results were catastrophic until the advent of more sophisticated anesthetic techniques, antibiotics, and fine surgical instruments, along with the pioneering work of Theodor Kocher [38]. William Halsted, Charles Mayo, and George Crile brought these European technical advances to the USA at the end of the nineteenth century to further refine the technique.

During the twentieth century, the incidence of thyroid cancer rose as imaging and fine-needle aspiration biopsies led to early detection of subclinical nodules [39]. As surgeons transitioned to operate on smaller glands, they began to push for less significant incisions in an effort to preserve the patient's cervical form and appearance. Initial methods included the use of endoscopes with gas insufflation to overcome the limited visualization provided by these smaller incisions [1•, 2]. They were, however, poorly received in the USA, as subplatysmal insufflation created a noncontained cavity within the neck. Also, subcutaneous emphysema, systemic absorption of carbon dioxide, and severe tachycardia were feared complications that were reported in the literature $[8,40]$. Conversely, in Southeast Asia, owing to the prevalence of nonesthetic scarring and the social stigmatization of young females with a visible scar, the technique was implemented, refined, and later enhanced with the advancement in robotic technology. In 2009, Kang et al. [19••] developed a robotic transaxillary technique for thyroid surgery, enabling the scar to be transferred to the less cosmetically sensitive axilla. Large patient series found this approach to have oncological outcomes equivalent to those of conventional thyroidectomy and result in less pain, with a faster return to functional activities $[17,19 \bullet \bullet]$. This remote-access approach has rapidly gained popularity in North American practice [41$44,45 \bullet$, 46]. However, this technique is clearly not minimally invasive and therefore requires compromise of some of the tremendous progress that has been made in thyroid surgery over the past several years.

Borrowing from minimally invasive, remote-access, robotic, and cosmetic principles, Terris et al. [18••] recently reported a hybrid technique, the robotic facelift approach. Robotic facelift thyroidectomy utilizes the benefit of robotic technology in combination with a familiar facelift incision approach to accomplish a remote-access thyroidectomy for patients who do not wish to have a neck scar. More recently, we reported the feasibility of performing a robotic transoral technique for accessing the central compartment of the neck and removing the thyroid in cadavers [16•], but there is no well-reported clinical experience with this approach.

\section{Indications and Patient Selection}

Despite the beautiful anatomic nature of thyroid surgery, the surgeon must be aware that many young female patients, with the exception of complications, will focus on incision length, location, design, and healing in the assessment of the overall quality of the surgery. Therefore, guiding principles that can serve as a framework for the safe implementation of these emerging technologies in thyroid surgery should be considered to avoid any unnecessary harm [45•]. Nonetheless, ideal patient selection criteria are not well established. The best candidates for this approach are small or average-sized (body mass index less than $30 \mathrm{~kg} / \mathrm{m}^{2}$ ) young patients with concerns of neck scarring or who have a history of keloid or hypertrophic scar formation. Nonetheless, our group reported a safe and feasible expansion of these selection criteria [36••]. In our experience with the transaxillary approach, $60 \%$ of our patients were overweight or obese, and the average nodule size was $2.5 \mathrm{~cm}$. We and others have also reported the feasibility of this approach in patients with 
well-differentiated thyroid cancer and cases of Graves's disease [29, 36••, 47]. Still, we believe that keeping the selection criteria conservative especially during the beginning of the surgeon's learning curve is vital for the safety and efficacy of robotic thyroid procedures. This approach is usually not offered to patients with a previous history of neck surgery or irradiation of the neck. Patients should also be screened for contraindications that affect patient positioning during this procedure, such as rotator cuff disease, shoulder/neck mobility problems, cervical spine disease, and previous neck, chest or axillary surgery. One potential drawback to the robotic facelift approach is that whereas total thyroidectomy can be performed through a single axillary incision, should total thyroidectomy be required, a bilateral facelift incision is necessary.

\section{Surgical Techniques}

\section{Robotic Transaxillary Thyroidectomy}

Most of the Western literature reports on robotic transaxillary thyroid surgery is limited to case series and small prospective and retrospective studies reporting surgical experience and outcome early in the initial learning curve, as defined by the American and Asian experiences [17, $36 \bullet, 48 \bullet$. Modifications to this approach were found to be necessary to accommodate the different operating dynamics of the Western patient population, to ensure this procedure can be safely replicated by US surgeons [36*0]. The current robotic surgical system has size limitations that hamper its use in the narrow confines of the head and neck. Furthermore, remote-access surgery can cause disorientation for the surgeon, as most head and neck surgeons are accustomed to wide visualization and the ability to see and control the major neurovascular structures in the head and neck. Eventually, doubts were raised that this procedure can be safely performed through a unilateral axillary incision in certain cases [43]. There is a significant learning curve for this approach. Our experience showed a learning curve of 45 cases [36•*], which is comparable to the Korean experience [17]. This, coupled with the technical demands this approach requires and the higher costs when compared with its conventional counterpart, tempered the enthusiasm of some surgeons [49•, 50].

This technique as described above includes flap creation under direct visualization to create a working space, docking the robotic system, and console operative time to perform the thyroid surgery. Patients are placed in a supine position under general anesthesia and intubated with an NIM endotracheal tube (Medtronic Xomed) to allow intraoperative monitoring of recurrent laryngeal nerve function. The neck is slightly extended and the arm ipsilateral to the lesion is placed cephalad and flexed above the head as described by Ikeda et al. [51]. An axillary incision is then made along the lateral border of the pectoralis major muscle. A subcutaneous flap is raised in a subplatysmal plane until the clavicle, and a window is then developed between the sternal and clavicular heads of the sternocleidomastoid muscle. A specially designed retractor is placed under the sternal head of the sternocleidomastoid and strap muscles, creating the work space superficial to the thyroid. Next, the da Vinci Si robot is docked from the side of the bed contralateral to the operative field, with the endoscope, Harmonic scalpel, and Maryland forceps entering via the axillary incision. An additional chest wall incision can be used in early experience to assist in dissection and manipulation of the thyroid gland. The dissection is performed mimicking the conventional cervical technique.

\section{Robotic Facelift (Postauricular-Occipital) Approach Thyroidectomy}

Terris et al. [18••] have recently described the feasibility of this technique in 14 patients using the posterior limb of a parotidectomy-facelift incision, which results in a decrease in the dissected surface area compared with the other remote-access techniques.

The incision is marked out adjacent to the postauricular crease, and crossing over to the occipital hairline at a position that will be obscured by the ear (Fig. 2). The patient is placed supine on the operating table. The head is turned $30^{\circ}$ away from the anticipated side of the lobectomy. The open dissection proceeds through a sequential identification of a series of structures starting with the sternocleidomastoid muscle. The dissection plane can be either superficial or deep to the platysma, and we prefer to stay in a superficial plane. The greater auricular nerve is then identified, and the dissection plane remains superficial to this nerve. The omohyoid is reflected ventrally to gain access to the superior pole of the thyroid gland. A customized retractor is introduced and placed underneath the strap muscles to maintain the operative pocket. An additional retractor is used (Greenberg retractor) to retract the sternocleidomastoid muscle and keep a wider operative field. The da Vinci robotic surgical system is then deployed. Three arms are usually used, compared with four arms in the transaxillary approach, because of the limited space. The camera arm is positioned first, holding a $30^{\circ}$ down endoscope parallel to the retractor system. A Maryland grasper is placed in the nondominant arm, and a Harmonic device is placed in the dominant arm (Video S2). The rest of the procedure is performed as described earlier for the transaxillary approach.

This technique has risks to the great auricular nerve and flap skin loss; however, none of these complications have 
Fig. 2 a The incision and dissection area for robotic facelift thyroidectomy. b The cosmetic results are valued by patients undergoing this approach
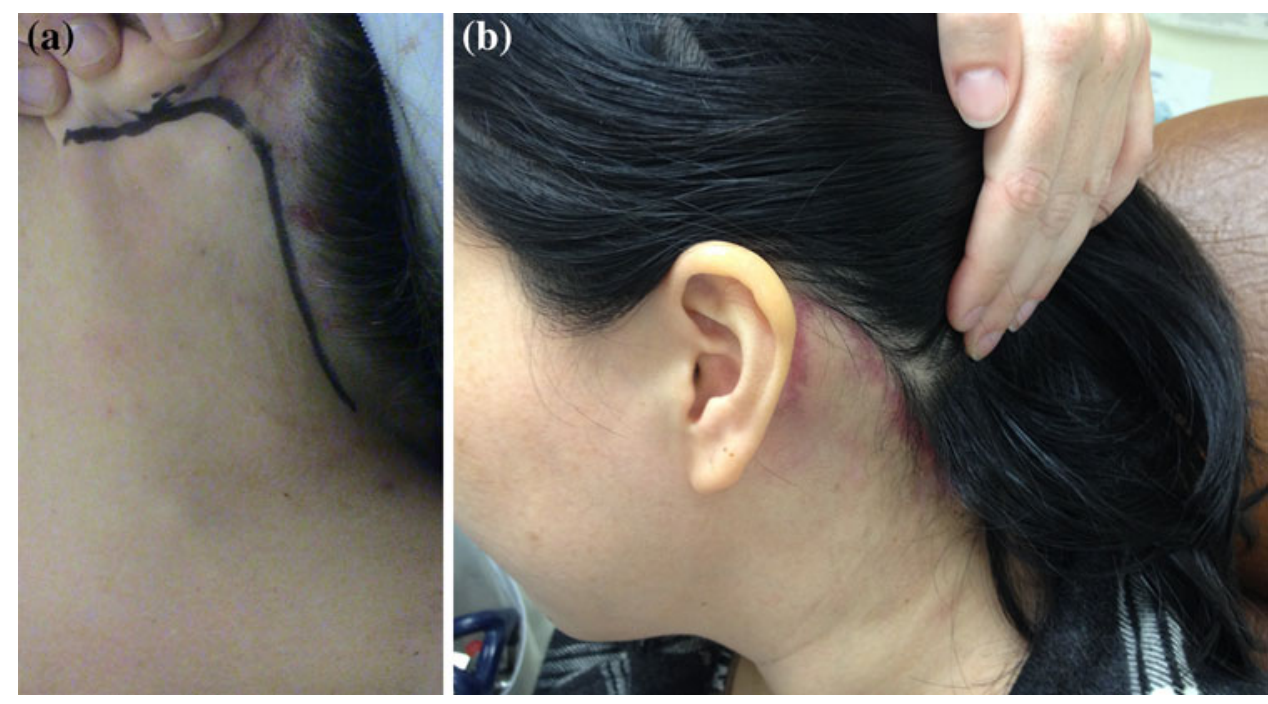

been reported. The main limitation of this approach is the inability to perform total thyroidectomy via a unilateral incision. Additional clinical experience is warranted to further validate this technique.

A group in Germany has recently reported a small series of patients in whom transoral video-assisted thyroidectomy was possible. This approach involves a $10-\mathrm{mm}$ sublingual sagittal incision, dissection through the floor of mouth musculature to the subplatysmal plane, and initial carbon dioxide insufflation, followed by bilateral $10-\mathrm{mm}$ vestibular incisions lateral to each mandibular canine [52]. Recently, we have reported the addition of robotic technology to this approach in human cadavers [16•]. However, this is still considered as an experimental technique, with a significantly reported conversion rate and complications.

\section{Complications}

It is well established that conventional cervical thyroid surgery has low morbidity with excellent outcomes. It is the gold standard approach for thyroidectomy, and it is essential that any new technique be evaluated in comparison with these current standards. A number of dramatic complications not associated previously with thyroid surgery have occurred. Published and unpublished reports of brachial plexopathy, esophageal perforation and transection, and high-volume blood loss have emerged. Additionally, prolonged paresthesia under the flap and muscle stiffness have been described by some patients. The arm positioning can cause overtraction and brachial plexus neuropraxia. This complication can be avoided by using somatosensory evoked potential response nerve monitoring in the transaxillary approach or the risk can be avoided completely in the retroauricular approach.

\section{Discussion}

Despite the advantages of robotic technology and the excellent results in terms of complication and cure rates, there are some concerns for its routine application in clinical practice. Experience of the entire surgical team with robotic technology is essential for optimizing outcomes using this procedure. Inserting and aligning the instruments requires a trained assistant and a team approach. We believe consistency of the team members, including operating room staff, yields the best improvements over time. In addition to mastering the technical aspects of the robotic surgical system, surgeons need to become familiar with the anatomic perspective of the lateral and posterior approaches to the thyroid and parathyroid glands.

The adoption of new technology in the operating room offers potential benefits as well as economic challenges. The need for specific instrumentation has been considered a source of additional costs compared with conventional surgery. Operative time, which was considered one of the limits of the technique, has been demonstrated to decrease with increasing experience.

\section{Conclusion}

Although it is important to examine new technologies and techniques, it is also essential that we implement them responsibly. Robotic surgery is still considered to be in the early stage of development, and presently robotic thyroidectomy remains more burdensome, lengthy, and costly. However, it is important to pursue these techniques in highvolume centers and evaluate the technology currently 
available in order to be prepared for future technology that may address today's limitations. What remains to be performed is a balanced investigation with rigorous data analysis to fully explore and recognize its advantages and limitations.

Disclosure Jeremy D. Richmon is a paid consultant for Intuitive Surgical. Ralph P. Tufano is a paid consultant for Medtronic Xomed and Ethicon. Salem I. Noureldine, Salah Mohamed, and Emad Kandil declare that they have no conflict of interest.

Compliance with Ethics Guidelines This article does not contain any studies with human or animal subjects performed by any of the authors.

\section{References}

Papers of particular interest, published recently, have been highlighted as:

- Of importance

- Of major importance

1. - Gagner M. Endoscopic subtotal parathyroidectomy in patients with primary hyperparathyroidism. Br J Surg. 1996;83(6):875. The concept of minimally invasive approaches to the thyroid and parathyroid glands considerably gained momentum in 1996 when Michelle Ganger performed a totally endoscopic subtotal parathyroidectomy for a man with familial hyperparathyroidism.

2. Huscher CS, Chiodini S, Napolitano C, Recher A. Endoscopic right thyroid lobectomy. Surg Endosc. 1997;11(8):877.

3. Ikeda Y, Takami H, Niimi M, Kan S, Sasaki Y, Takayama J. Endoscopic thyroidectomy by the axillary approach. Surg Endosc. 2001;15(11):1362-4.

4. Gagner M, Inabnet WB 3rd. Endoscopic thyroidectomy for solitary thyroid nodules. Thyroid. 2001;11(2):161-3.

5. Miccoli P. Minimally invasive surgery for thyroid and parathyroid diseases. Surg Endosc. 2002;16(1):3-6.

6. Cho YU, Park IJ, Choi KH, Kim SJ, Choi SK, Hur YS, et al. Gasless endoscopic thyroidectomy via an anterior chest wall approach using a flap-lifting system. Yonsei Med J. 2007;48(3):480-7.

7. Bellantone R, Lombardi CP, Raffaelli M, Rubino F, Boscherini M, Perilli W. Minimally invasive, totally gasless video-assisted thyroid lobectomy. Am J Surg. 1999;177(4):342-3.

8. Kang SW, Jeong JJ, Yun JS, Sung TY, Lee SC, Lee YS, et al. Gasless endoscopic thyroidectomy using trans-axillary approach; surgical outcome of 581 patients. Endocr J. 2009;56:361-9.

9. Sasaki A, Nakajima J, Ikeda K, Otsuka K, Koeda K, Wakabayashi G. Endoscopic thyroidectomy by the breast approach: a single institution's 9-year experience. World J Surg. 2008;32(3): $381-5$.

10. Lee DI. Robotic prostatectomy: what we have learned and where we are going. Yonsei Med J. 2009;50(2):177-81.

11. Advincula AP, Song A. The role of robotic surgery in gynecology. Curr Opin Obstet Gynecol. 2007;19:331-6.

12. Nifong LW, Chitwood WR, Pappas PS, Smith CR, Argenziano M, Starnes VA, et al. Robotic mitral valve surgery: a United States multicenter trial. J Thorac Cardiovasc Surg. 2005;129: $1395-404$.
13. Hollands CM, Dixey LN. Robotic-assisted esophagoesophagostomy. J Pediatr Surg. 2002;37:983-5; discussion 985.

14. Patel VR. Essential elements to the establishment and design of a successful robotic surgery programme. Int J Med Robot. 2006;2(1):28-35.

15. Moore EJ, Olsen KD, Kasperbauer JL. Transoral robotic surgery for oropharyngeal squamous cell carcinoma: a prospective study of feasibility and functional outcomes. Laryngoscope. 2009;119(11): 2156-64.

16. • Richmon JD, Holsinger FC, Kandil E, Moore MW, Garcia JA, Tufano RP. Transoral robotic-assisted thyroidectomy with central neck dissection: preclinical cadaver feasibility study and proposed surgical technique. J Robot Surg. 2011;5(4):279-82. This novel approach through the floor of the mouth is truly cosmetic and probably painless.

17. Lee J, Yun JH, Nam KH, Soh EY, Chung WY. The learning curve for robotic thyroidectomy: a multicenter study. Ann Surg Oncol. 2011;18(1):226-32.

18. $\bullet$ Terris DJ, Singer MC, Seybt MW. Robotic facelift thyroidectomy: II. Clinical feasibility and safety. Laryngoscope. 2011;121(8):1636-41. Terris et al. described the feasibility of this techniqu using the posterior limb of a parotidectomy-facelift incision, which results in a decrease in the dissected surface area compared with the other remote-access techniques

19. •• Kang S-W, Jeong J, Yun J-S, Sung T, Lee S, Lee Y, et al. Robot-assisted endoscopic surgery for thyroid cancer: experience with the first 100 patients. Surg Endosc. 2009(11):2399-406. Owing to the prevalence of nonesthetic scarring and the social stigmatization of young females with visible scars in South Korea, Kang et al. developed a robotic transaxillary gasless technique for thyroid surgery.

20. Lee KE, Rao J, Youn YK. Endoscopic thyroidectomy with the da Vinci robot system using the bilateral axillary breast approach (BABA) technique: our initial experience. Surg Laparosc Endosc Percutan Tech. 2009;19:e71-5.

21. Tolley N, Arora A, Palazzo F, Garas G, Dhawan R, Cox J, et al. Robotic-assisted parathyroidectomy: a feasibility study. Otolaryngol Head Neck Surg. 2011;144:859-66.

22. Kang SW, Jeong JJ, Nam KH, Chang HS, Chung WY, Park CS. Robot-assisted endoscopic thyroidectomy for thyroid malignancies using a gasless transaxillary approach. J Am Coll Surg. 2009;209:e1-7.

23. Palazzo FF, Delbridge LW. Minimal-access/minimally invasive parathyroidectomy for primary hyperparathyroidism. Surg Clin North Am. 2004;84:717-34.

24. • Tibblin S, Bondeson AG, Bondeson L, Ljungberg O. Surgical strategy in hyperparathyroidism due to solitary adenoma. Ann Surg. 1984;200(6):776-84. This was the first unilateral approach for solitary parathyroid adenoma resection.

25. Miccoli P, Bendinelli C, Berti P, Vignali E, Pinchera A, Marcocci C. Video-assisted versus conventional parathyroidectomy in primary hyperparathyroidism: a prospective randomized study. Surgery. 1999;126:1117-21; discussion 1121-2.

26. Costello D, Norman J. Minimally invasive radioguided parathyroidectomy. Surg Oncol Clin N Am. 1999;8(3):555-64.

27. Katz L. Abdel Khalek M, Crawford B, Kandil E. Robotic-assisted transaxillary parathyroidectomy of an atypical adenoma. Minim Invasive Ther Allied Technol. 2011;21(3):201-5.

28. Landry CS, Grubbs EG, Morris GS, Turner NS, Holsinger FC, Lee JE, et al. Robot assisted transaxillary surgery (RATS) for the removal of thyroid and parathyroid glands. Surgery. 2011; 149:549-55.

29. Foley CS, Agcaoglu O, Siperstein AE, Berber E. Robotic transaxillary endocrine surgery: a comparison with conventional open technique. Surg Endosc. 2012;26(8):2259-66. 
30. Tanna N, Joshi AS, Glade RS, Zalkind D, Sadeghi N. Da Vinci robot-assisted endocrine surgery: novel applications in otolaryngology. Otolaryngol Head Neck Surg. 2006;135:633-5.

31. Noureldine S, Lewing N, Emad K. The role of the roboticassisted transaxillary gasless approach for the removal of parathyroid adenomas. ORL. In press 2013.

32. Bodner J, Profanter C, Prommegger R, Greiner A, Margreiter R, Schmid T. Mediastinal parathyroidectomy with the da Vinci robot: presentation of a new. J Thorac Cardiovasc Surg. 2004;127(6):1831-2.

33. Timmerman GL, Allard B, Lovrien F, Hickey D. Hyperparathyroidism: robotic-assisted thoracoscopic resection of a supernumary. J Laparoendosc Adv Surg Tech A. 2008;18(1):76-9.

34. Ismail $M$, Maza $S$, Swierzy $M$, Tsilimparis $N$, Rogalla $P$, Sandrock D, et al. Resection of ectopic mediastinal parathyroid glands with the da Vinci robotic. Br J Surg. 2010;97(3): 337-43.

35. Chen H, Pruhs Z, Starling JR, Mack E. Intraoperative parathyroid hormone testing improves cure rates in patients undergoing minimally invasive parathyroidectomy. Surgery. 2005;138(4):583-7; discussion 587-90.

36. • Kandil EH, Noureldine SI, Yao L, Slakey DP. Robotic transaxillary thyroidectomy: an examination of the first one hundred cases. J Am Coll Surg. 2012;214(4):558-64. This is the largest experience in the USA with robotic transaxillary thyroid surgery. The authors examine the effect of experience to determine the learning curve and improvements over time in operative performance.

37. Li X, Massasati SA, Kandil E. Single incision robotic transaxillary approach to perform parathyroidectomy. Gland Surg. 2012;1(3):169-70. doi:10.3978/j.issn.2227-684X.2012.09.02.

38. Kocher T. Zur pathologie und therapie des kropfes. Dtsch Z Chirug. 1874;4:417.

39. Enewold L, Zhu K, Ron E, Marrogi AJ, Stojadinovic A, Peoples GE, et al. Rising thyroid cancer incidence in the United States by demographic and tumor characteristics, 1980-2005. Cancer Epidemiol Biomarkers Prev. 2009;18:784-91.

40. Bellantone R, Lombardi CP, Rubino F, Perilli V, Sollazzi L, Mastroianni G, et al. Arterial PCO2 and cardiovascular function during endoscopic neck surgery with carbon dioxide insufflation. Arch Surg. 2001;136:822-7.

41. Kuppersmith RB, Holsinger FC. Robotic thyroid surgery: an initial experience with North American patients. Laryngoscope. 2011;121(3):521-6.
42. Berber E, Siperstein A. Robotic transaxillary total thyroidectomy using a unilateral approach. Surg Laparosc Endosc Percutan Tech. 2011;21:207-10.

43. Landry CS, Grubbs EG, Perrier ND. Bilateral robotic-assisted transaxillary surgery. Arch Surg. 2010;145:717-20.

44. Kandil E, Winters R, Aslam R, Friedlander P, Bellows C. Transaxillary gasless robotic thyroid surgery with nerve monitoring: initial 2 experience in a North American center. Minim Invasive Ther Allied Technol. 2011;21(2):90-5.

45. - Perrier ND, Randolph GW, Inabnet WB, Marple BF, VanHeerden J, Kuppersmith RB. Robotic thyroidectomy: a framework for new technology assessment and safe implementation. Thyroid. 2010;20(12):1327-32. As for the introduction of new technology to any surgery, a framework for the safe implementation of this technique has been outlined to avoid any unnecessary harm to patients, surgeons, and society.

46. Aliyev S, Taskin HE, Agcaoglu O, Aksoy E, Milas M, Siperstein A, et al. Robotic transaxillary total thyroidectomy through a single axillary incision. Surgery. 2013;153(5):705-10.

47. Kandil E, Noureldine S, Abdel Khalek M, Alrasheedi S, Aslam $\mathrm{R}$, Friedlander $\mathrm{P}$, et al. Initial experience using robot- assisted transaxillary thyroidectomy for Graves' disease. J Visc Surg. 2011;148:e447-51.

48. - Jackson NR, Yao L, Tufano RP, Kandil EH. Safety of robotic thyroidectomy approaches: meta-analysis and systematic review. Head Neck. 2013. doi:1002/hed.23223. This is a meta-analysis suggesting that robotic thyroidectomy is as safe, feasible, and efficacious as conventional cervical and endoscopic thyroidectomy.

49. - Perrier ND. Why I have abandoned robot-assisted transaxillary thyroid surgery. Surgery. 2012;152(6):1025-6. The technical demands the robotic approach requires and the higher costs when compared with its conventional counterpart tempered the enthusiasm of some surgeons in the West.

50. Inabnet WB 3rd. Robotic thyroidectomy: must we drive a luxury sedan to arrive at our destination safely? Thyroid. 2012;22(10): 988-90.

51. Ikeda Y, Takami H, Niimi M, Kan S, Sasaki Y, Takayama J. Endoscopic thyroidectomy and parathyroidectomy by the axillary approach. A preliminary report. Surg Endosc. 2002;16(1):92-5.

52. Benhidjeb T, Wilhelm T, Harlaar J, Kleinrensink GJ, Schneider TA, Stark M. Natural orifice surgery on thyroid gland: totally transoral video-assisted thyroidectomy (TOVAT): report of first experimental results of a new surgical method. Surg Endosc. 2009;23(5):1119-20. 